\title{
El poblado de La Codera. Aproximación al urbanismo de la I Edad del Hierro
}

\section{The settlement of La Codera. Approach to urbanism of the Early Iron Age}

\author{
FÉLIX J. Montón BROTO*
}

\section{RESUMEN}

Después de considerar el emplazamiento del poblado y establecer las fases de ocupación, se hace una descripción de los elementos urbanísticos que forman el asentamiento, enumerando los equipamientos del poblado, así como los elementos que forman parte de las estructuras domésticas. Se especifican las vías de circulación, usos del espacio y fortificaciones.

\section{PALABRAS CLAVE}

Edad del Hierro, urbanismo, fortificaciones, cisterna, valle del Ebro.

\section{ABSTRACT}

After having considered the location of the site and having established the different phases of settlement, a description of the urbanism is done, describing different urban equipaments, as well, as different constructuve elements within the domestic structures. The ways of transit within the settlement, the defensive system and uses of space are commented.

KEY WORDS

Iron Age, urbanism, defenses, cistern, Ebro valley.

\section{INTRODUCCIÓN}

Con el presente trabajo quiero contribuir al recuerdo de nuestra compañera Victoria aportando algunas novedades procedentes del trabajo de investigación derivado de la excavación arqueológica del poblado de La Codera, al que la UNED contribuye con los medios económicos dispuestos por el Centro de Barbastro,

* UNED, Barbastro 
con la aprobación del Departamento y con la colaboración de nuestros alumnos de varios centros asociados de España. Justo es decir que los trabajos son también sufragados por el Gobierno de Aragón, el Ayuntamiento de Alcolea de Cinca y la empresa EUROPAC. Igualmente hemos contado con la asistencia de alumnos universitarios de otras universidades españolas, especialmente de la Universidad de Zaragoza.

El poblado de La Codera forma parte de un interesante conjunto arqueológico que contiene en un espacio de $1 \mathrm{~km}$ escaso de radio un poblado de la Edad del Bronce con su correspondiente necrópolis, un asentamiento de Campos de Urnas, un poblado de la Edad del Hierro y dos necrópolis asociadas a él, un poblado ibérico, una necrópolis de época incierta y restos de poblamiento protohistórico dispersos en curso de estudio. En estos momentos conocemos mucho mejor el desarrollo del poblamiento de la zona a lo largo de más de un milenio. En efecto, al establecimiento más antiguo correspondiente a un momento indeterminado del Bronce Medio o Final I (Poblado del Bronce, sin excavar) sucede un asentamiento en ladera, hacia el Bronce Final II o III (Poblado de Campos de Urnas, excavado parcialmente). Posteriormente se produce la erección en altura del hábitat fortificado de la I Edad del Hierro, al menos con dos fases (Poblado del Hierro), para finalizar con la construcción del poblado de época ibérica (Poblado Ibérico). Estos vestigios abarcan aproximadamente un milenio, entre 1.200 y 200 a.C, aparentemente con pocas interrupciones, convirtiéndolos en un campo de estudio único en Aragón para el conocimiento de estas etapas protohistóricas.

Algunos de estos yacimientos ya son conocidos desde hace varias décadas y de ellos dan referencia R. Pita, J.L. Maya y la Carta Arqueológica de Huesca. En 1982 se efectuó la excavación de uno de los túmulos encontrándose los materiales en el Museo de Huesca. Desde 1997 se vienen desarrollando ininterrumpidamente sucesivas campañas incluidas en los Planes de Investigación del Gobierno de Aragón a través del aval del Museo de Huesca. Las siguientes líneas son una primicia sobre los elementos urbanísticos que el estudio en profundidad del poblado de la Edad del Hierro deberá aportar a este campo de conocimiento. Omitimos por tanto los análisis pormenorizados, las referencias específicas y los temas relativos a la construcción propiamente dicha, es decir, materiales, técnicas, sistemas constructivos, aparejos o medidas.

\section{CONSIDERACIONES URBANÍSTICAS}

No es tarea fácil acometer el estudio del urbanismo durante la I Edad del Hierro en algunas partes de la Península lbérica dado el corto número de yacimientos excavados y la confusión existente entre la terminología utilizada para caracterizar este periodo de la protohistoria peninsular. En el Valle Medio del Ebro el problema se agudiza al participar de las influencias celtibéricas en su parte occidental y las aportaciones de la cultura de los campos de urnas en su sector oriental. La cues- 


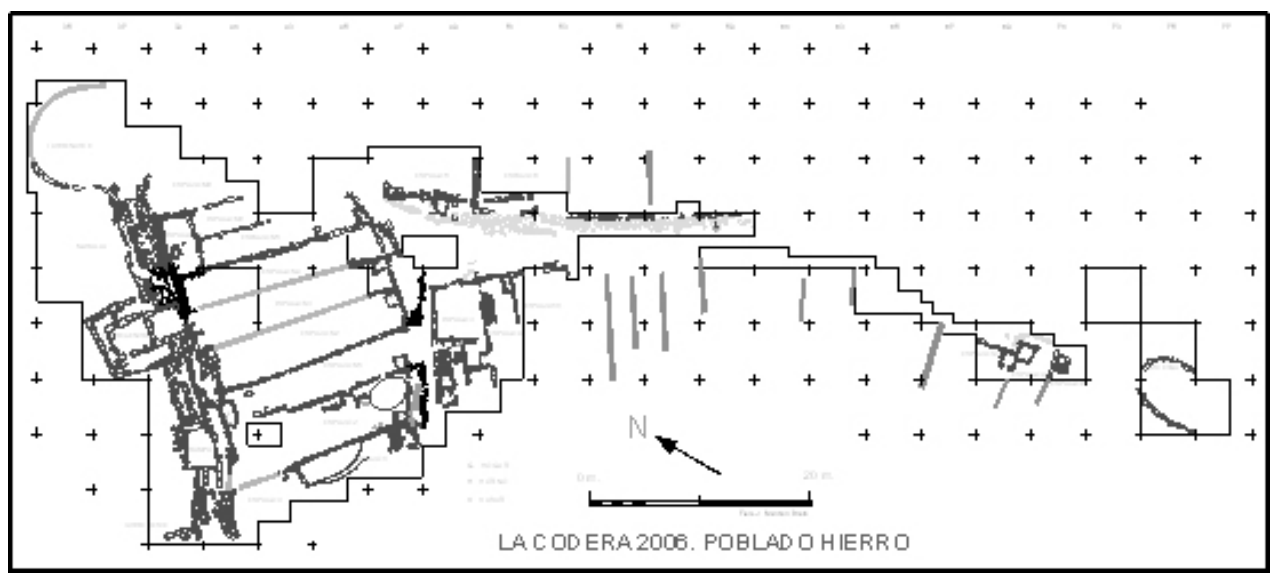

Fig. 1. Estructuras excavadas hasta 2006.

tión se complica al hacer su penetración la cultura ibérica y el resultado es que, tras caracterizar perfectamente los elementos ibéricos, sus directos antecesores se identifican con el llamado Bronce Final en las regiones orientales y con una imprecisa transformación del horizonte céltico o celtibérico hacia el mundo ibérico pleno en las zonas más próximas a la meseta. En consecuencia y por lo que hace referencia a nuestra zona de estudio se tiende a ignorar la existencia de una I Edad del Hierro o a hacerla coincidir con la fase III del citado Bronce Final.

La realidad es que contamos con muy pocos ejemplos en los que basar nuestras investigaciones. El poblado de Vilars, en Arbeca y el del Cabezo de la Cruz, en La Muela, son los únicos excavados hasta el momento y que pertenecen a la época que llamamos I Edad del Hierro. Sin pretender entrar en cuestiones cronológicas, que nos llevarían a tratar de compaginar las diferentes propuestas secuenciales planteadas por diversos investigadores en los últimos 25 años y que serían objeto de otro artículo, únicamente hago constar que las dataciones radiocarbónicas disponibles nos sitúan el poblado de La Codera en una horquilla que va de finales del s.VII (2570 $\pm 60 \mathrm{GrN}-26053)$ a principios del V a.C. $(2460 \pm 35$ GrA-24400).

\subsection{El emplazamiento}

La situación del poblado viene determinada por sus cualidades estratégicas. Se ubica en un punto en que los cauces de los ríos Cinca y Alcanadre distan apenas tres kilómetros, próximo al encuentro de ambos y controlando sus cursos, dominando visualmente un amplio territorio que va desde las cumbres pirenaicas hasta las cercanías del encuentro entre el Segre, el Cinca y el Ebro. El lugar elegido para la construcción del asentamiento es un espolón que forma parte de las 

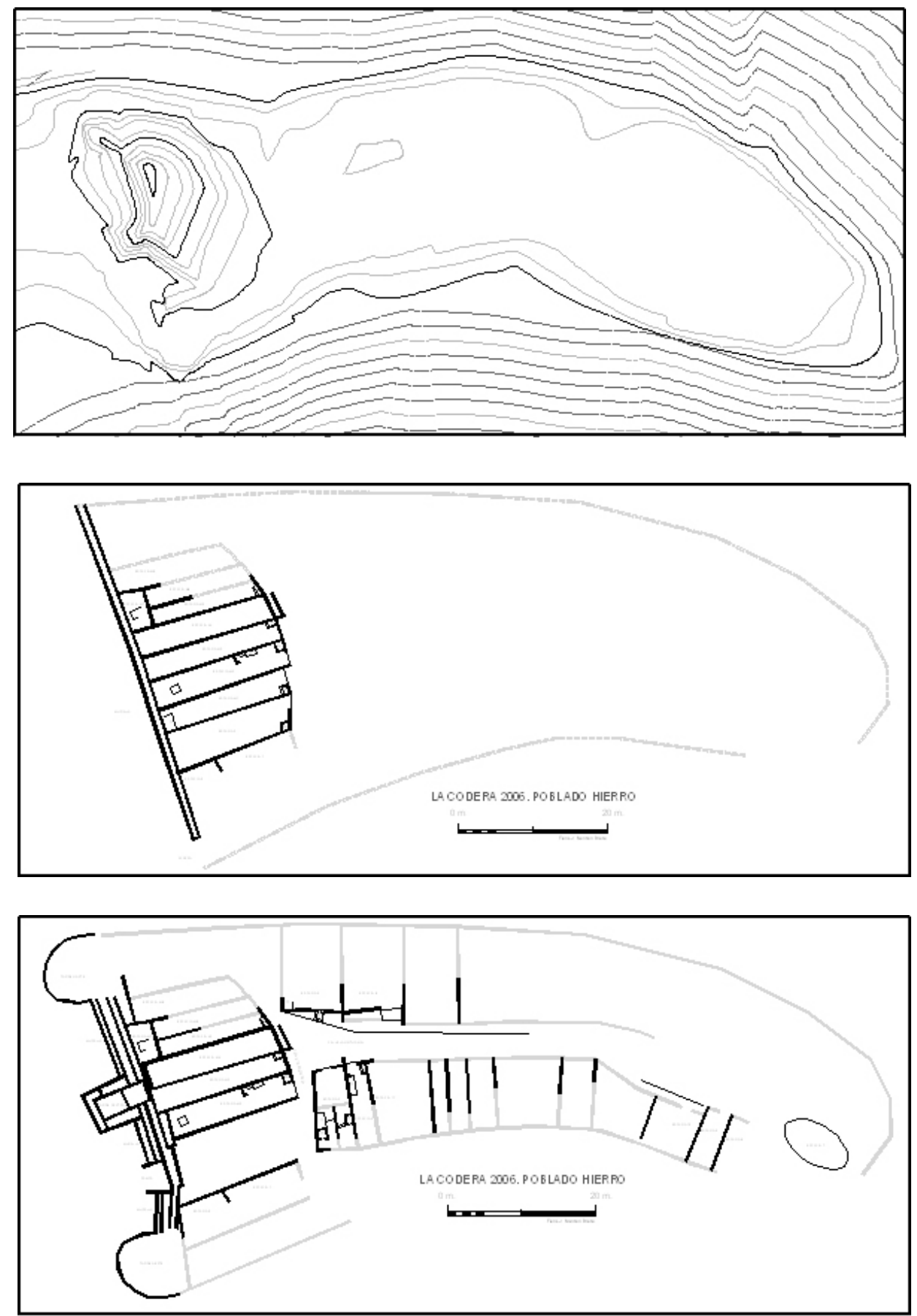

Fig. 2. Topografía previa al asentamiento y etapas constructivas. 
terrazas de la margen derecha del río Cinca. Tiene una forma ligeramente arriñonada y está rodeado de fuertes pendientes en todo su perímetro salvo en el lado noroeste que lo comunica con una extensión de terreno llano, única parte de cómodo acceso. A unos 100 metros del extremo sureste una pequeña elevación de unos tres metros sirve como pequeña defensa natural y delimita la zona construida a la par que vale de apoyo a las defensas del poblado. En su primera fase, esta elevación sirvió de referencia para la erección de un muro de defensa tras el cual se construirían las primeras habitaciones. Posteriormente este muro será reforzado y a las habitaciones adosadas a la muralla se añadirá un conjunto de estancias a lo largo del eje mayor del asentamiento. Al mismo tiempo se cambiará la situación de acceso al poblado, teniendo que amortizar algunas de las estructuras de la primera fase.

El resultado es que el asentamiento adopta la forma del lugar en que se levanta, condicionado por el límite de las pendientes y la pequeña elevación que le sirve de separación de la parte llana. Del mismo modo las vías de circulación se adaptan al terreno: una calle, que hemos llamado transversal, ligeramente curvada siguiendo las fachadas de las habitaciones que se adosan a la muralla y otra calle, denominada longitudinal, que tuerce siguiendo la forma del espolón. Igualmente las construcciones adosadas a la muralla se levantan escalonadas de acuerdo con la citada elevación previa a la construcción del hábitat y la calle transversal mantiene la pendiente original del terreno. Hemos podido identificar restos de un camino casi desaparecido, en la vertiente occidental de poblado, que parece corresponder al antiguo itinerario de acceso a la parte llana, ante las puertas del poblado.

\subsection{Distribución del hábitat: usos del mismo}

De acuerdo con las técnicas constructivas, el reparto de los materiales arqueológicos recuperados y el tamaño y situación de los espacios podemos proponer la siguiente atribución de usos dentro del poblado. En primer lugar espacios de habitación identificados por determinadas estructuras domésticas que veremos más adelante y por la abundancia de material cerámico correspondiente a vajilla casera. Un segundo grupo de espacios destinados a la actividad artesanal, caracterizados por la escasa presencia de material arqueológico y la presencia de estructuras singulares como enlosados y hornos. En tercer lugar las vías de circulación anteriormente citadas y a las que se abren todos los espacios pertenecientes a los dos grupos ya mencionados. Por último es probable la existencia de un espacio abierto, libre de construcciones, situado al fondo del poblado y que pudo servir como refugio al ganado. 


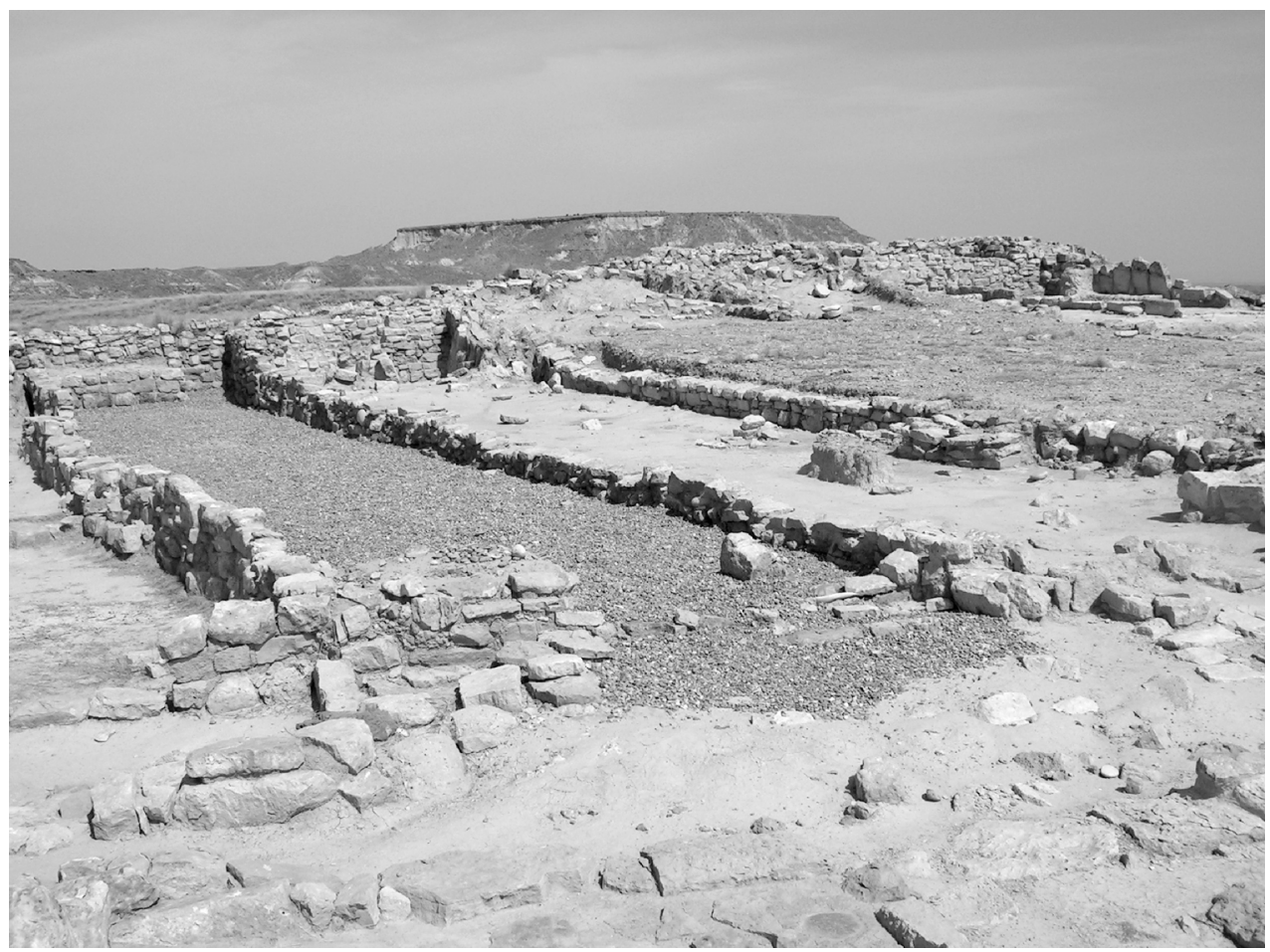

Fig. 3. Habitaciones adosadas a la muralla. Primera fase constructiva.

\subsection{Elementos urbanísticos}

\subsubsection{La casa}

Bajo este apelativo denominamos a las estructuras diferenciadas, que constituyen una unidad y que se abren a los espacios de circulación. De lo excavado hasta el momento podemos establecer dos tipos básicos. El primero, la casa vivienda, de planta alargada rectangular y acondicionada con algunos elementos complementarios como tabiques internos, vasares o bancos y hogares. Obviamente la presencia de ajuares cerámicos de cocina y servicio y elementos de uso doméstico o adorno asegura la identificación de la función del espacio. El segundo tipo es la casa-lugar de trabajo, de planta mucho menos alargada, provista de estructuras de transformación y de almacenamiento, como hornos y enlosados, o con presencia significativa de objetos de trabajo, en este caso molinos. La ausencia de elementos pertenecientes al servicio doméstico o al uso personal completa la diferencia con el primer tipo.

Al tipo casa-vivienda pertenecen los espacios M1, M5, M6, M7 y M8, excavados totalmente, los E2 y M2, excavados parcialmente y los M3 y M4 todavía por excavar. El tipo casa-lugar de trabajo está representado por los espacios E3 y E5. 

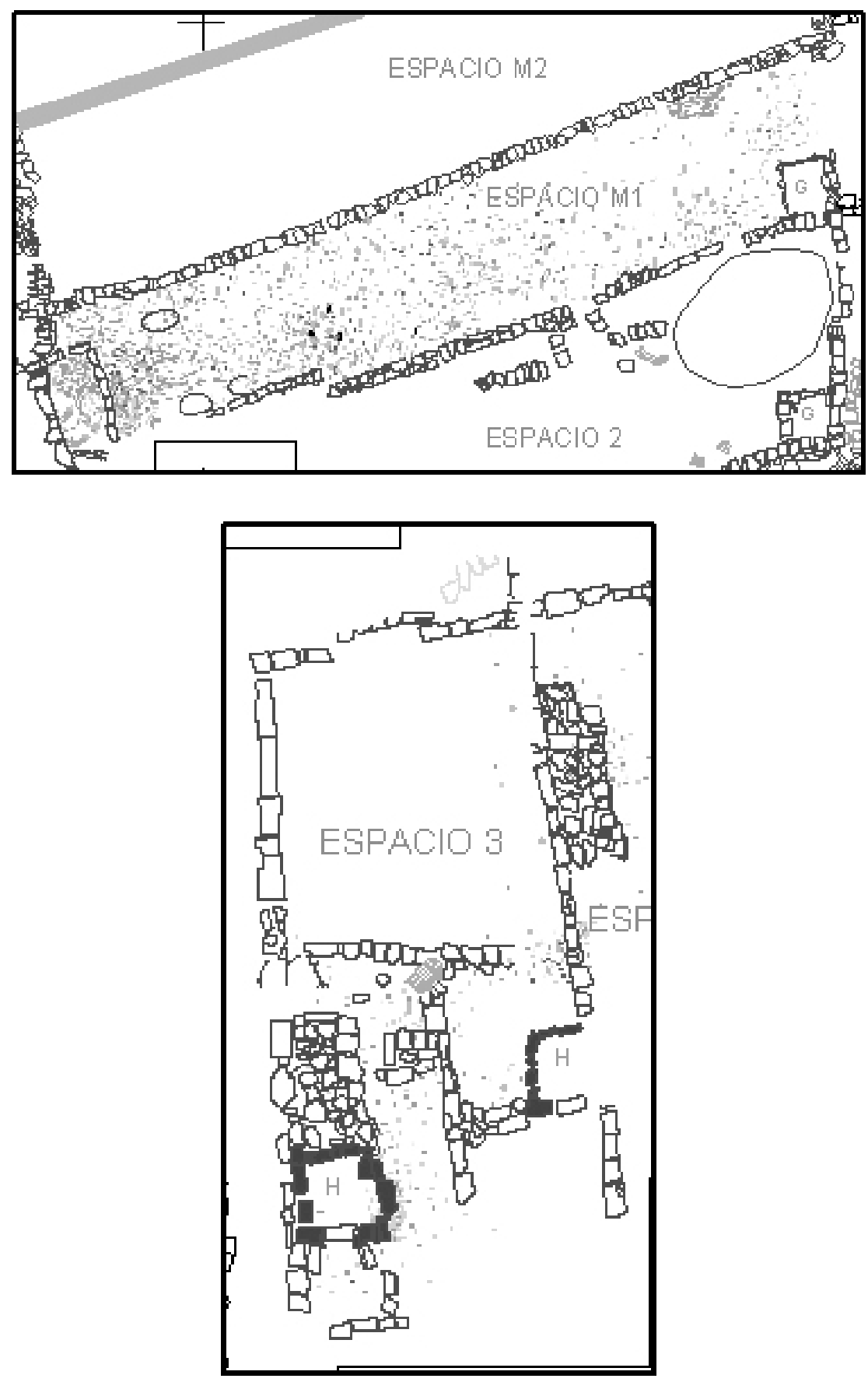

Fig. 4. Comparación de la densidad de materiales entre una vivienda (M1) $y$ un espacio de trabajo (E3). 


\subsubsection{Estructuras singulares. La cisterna}

Llamado en principio espacio E7, en el momento de su aparición, se trata de una estructura de planta elíptica cuyos ejes miden 5 y 7'80 metros respectivamente. Se construyó sobre la roca natural levantando las paredes del vaso sobre el estrato natural de caliza en el que descansa el asentamiento, uno de los muchos que componen la formación geológica del paraje. El aparejo, con un acusado talud hacia el interior, está formado por sillares de tamaño medio, en su mayoría de forma alargada. La profundidad debió aproximarse a los dos metros, conservándose $1^{\prime} 50 \mathrm{~m}$. en su parte más alta y habiendo perdido dos tercios de la estructura en su parte más erosionada, hacia el sur. El fondo es prácticamente horizontal formado por el citado estrato de caliza y aparentemente no tuvo ningún tipo de revestimiento. El pretil pudo estar rematado de adobe, de materia lígnea, o una combinación de ambas, a juzgar por los restos de estos materiales observados en la estratigrafía del interior. Como es habitual en estas estructuras, su contenido estaba formado por cuantiosos restos óseos pertenecientes a una variada fauna tanto doméstica como salvaje de ovicápridos, suidos, équidos, cérvidos y bóvidos. Igualmente considerables restos cerámicos correspondientes a formas variadas y entre el material lítico destacan un gran número de canas de diferentes tamaños.

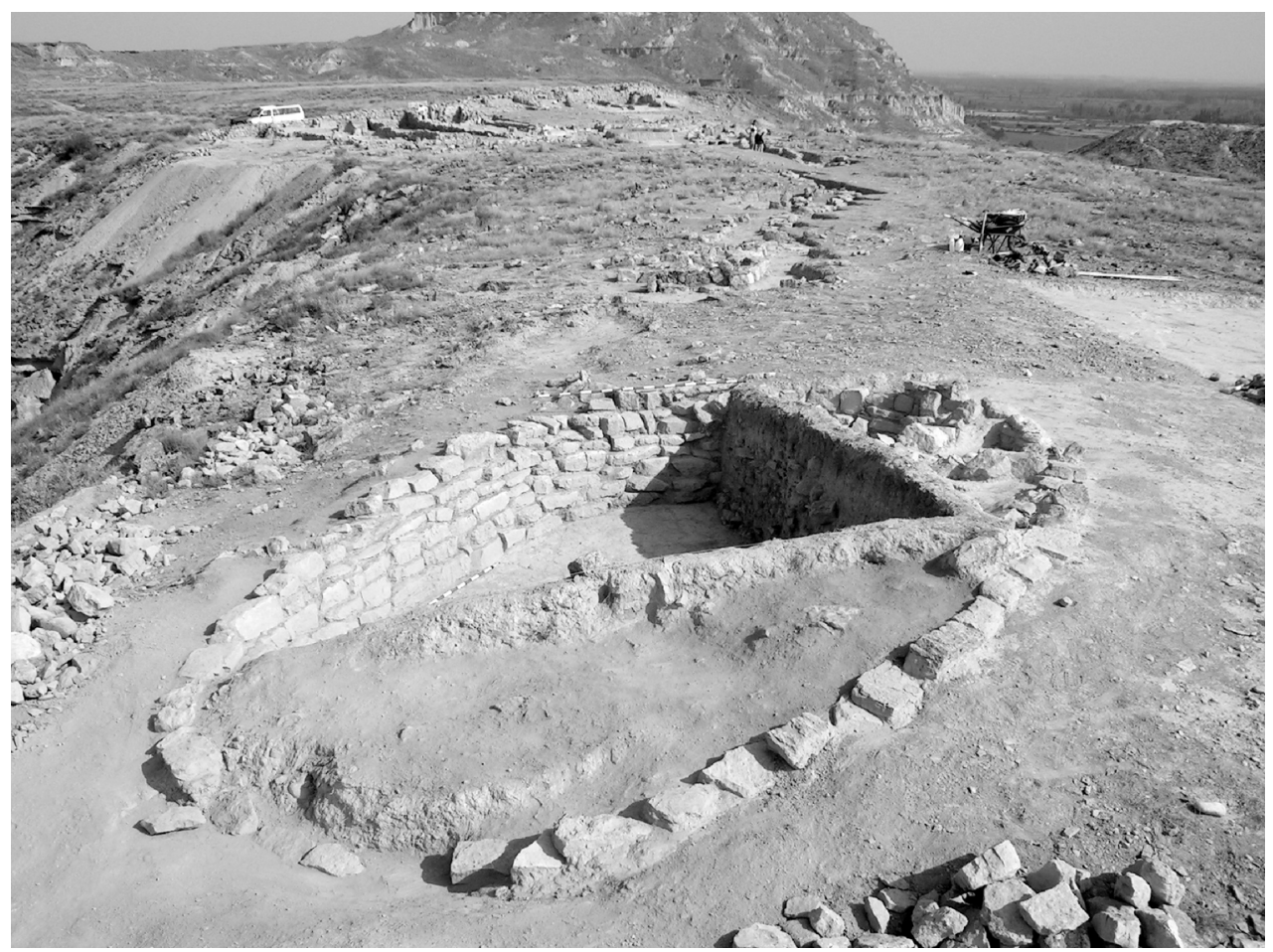

Fig. 5. Panorámica del poblado con la cisterna en primer plano. 


\subsubsection{Infraestructuras urbanas. Aceras y porches.}

Entre los complementos del equipamiento urbanístico del poblado hemos podido documentar la existencia de aceras en la parte correspondiente a la segunda fase de la ocupación, así como voladizos en las techumbres que proporcionan espacios cubiertos, ya sea como zonas de trabajo al aire libre o como zonas de descanso donde protegerse de la lluvia o del sol.

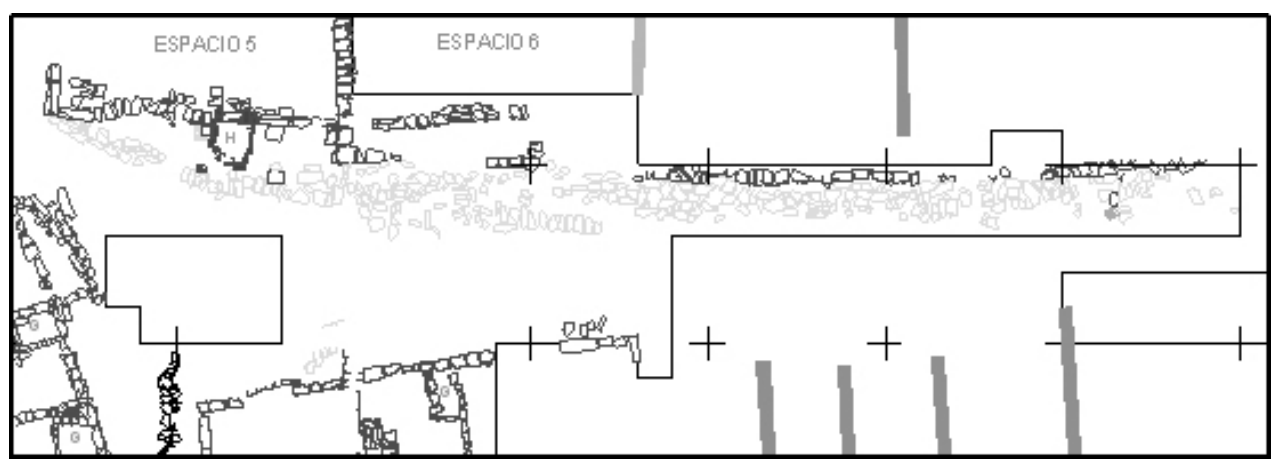

Fig. 6. Detalle de la calle longitudinal y aceras.

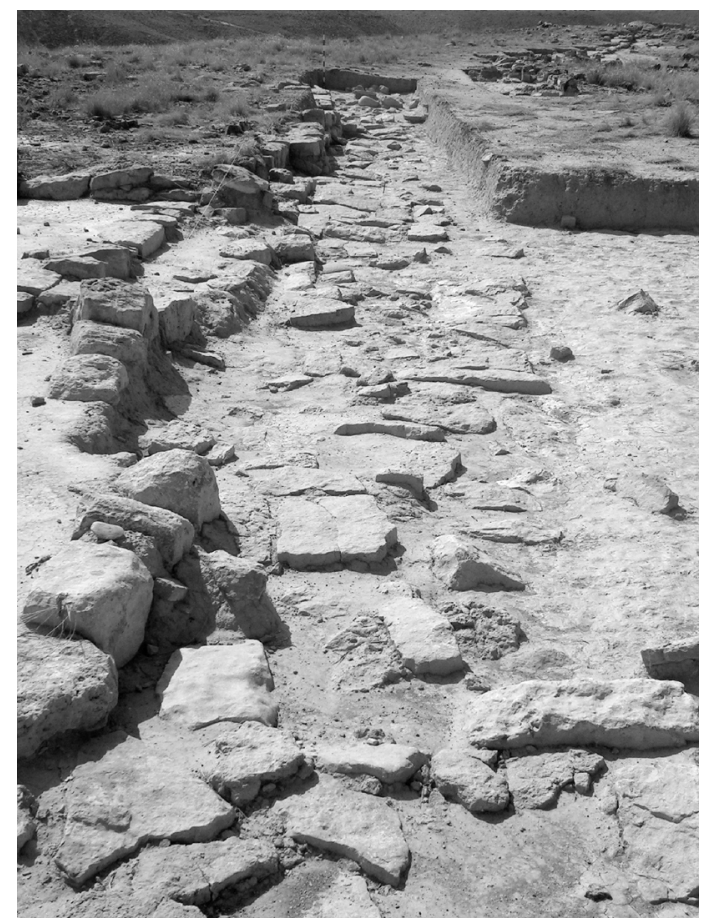

Fig. 7. La acera del lado oriental de la calle longitudinal. 
Las aceras se encuentran a lo largo de las fachadas de las construcciones de la calle longitudinal, conservándose mejor en la parte oriental que en la occidental. Están formadas por un bordillo de lajas de mediano tamaño rellenándose su interior en ocasiones con tierra apisonada aunque en el lado oriental de la calle están compuestas por un enlosado en su totalidad. La anchura es variable teniendo una media de unos dos metros y de momento la longitud documentada hasta hoy es de 35 metros, que corresponden a la parte excavada de las habitaciones situadas al este de la calle longitudinal. Aparentemente toda la calle estuvo provista de este equipamiento, aunque hay que esperar a la excavación total del espacio para confirmarlo.

Por lo que respecta a los porches, su existencia se desprende de la existencia de muros medianiles que se prolongan más allá de las fachadas y que, a modo de antas, sirven de apoyo a un voladizo de la techumbre que actúa como un porche. Así se comprueba en el muro que separa el espacio E5 del E6 y de éste con E7; lo mismo para el medianil entre E3 y E4. Ante el espacio E5 y flanqueando el horno adosado a este espacio se conservan cuatro sillares que evidentemente sirvieron de apoyo a otros tantos postes que sostuvieron sin duda esta parte del tejado.

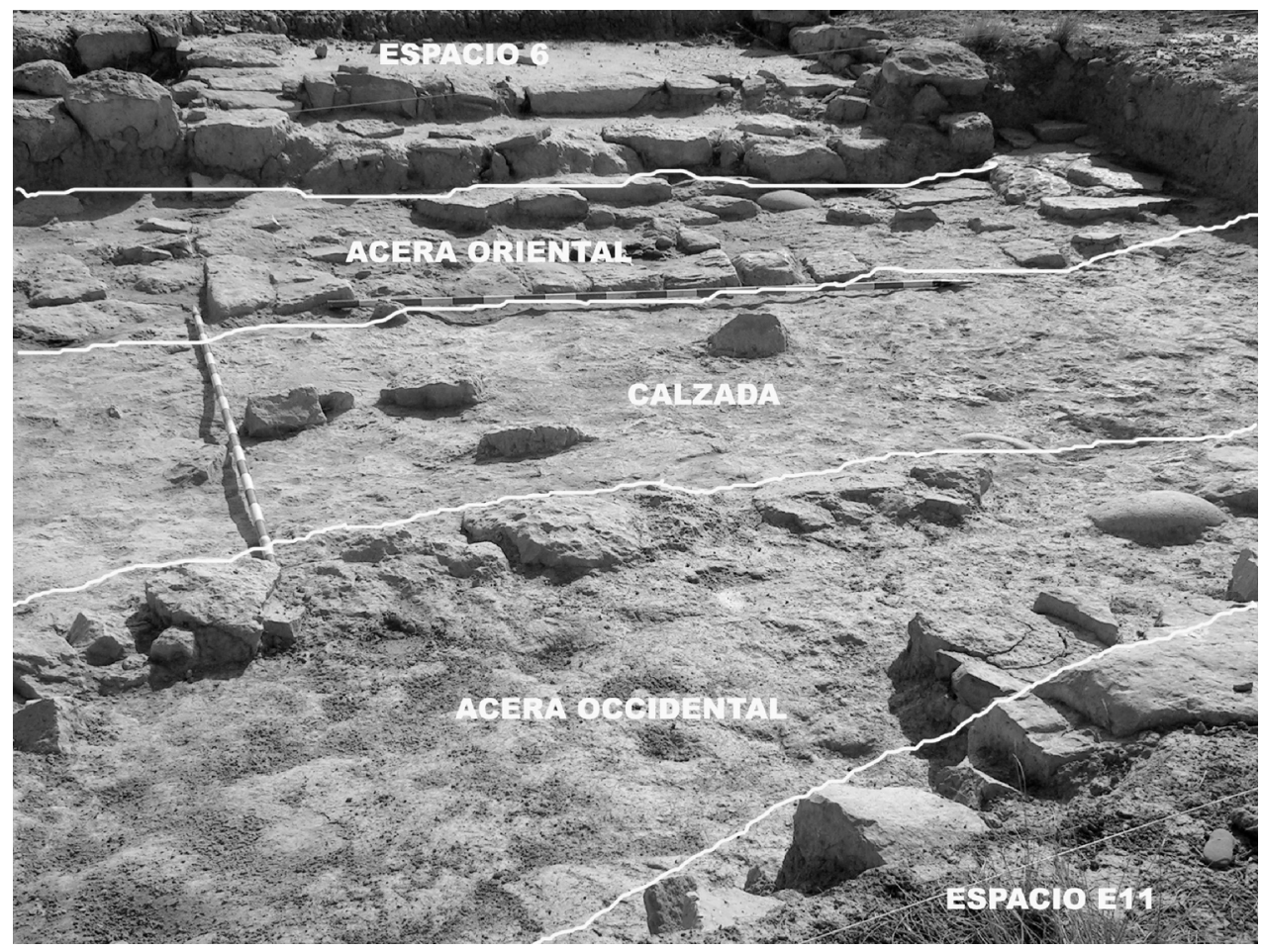

Fig. 8. Espacio de circulación entre las casas con la calzada y las aceras. 


\subsubsection{Infraestructuras domésticas}

Aunque la estructura interna de las edificaciones es muy simple y habida cuenta de que se han perdido construcciones hechas con materiales perecederos (madera, cañas, adobes, tapial, tejidos), sin embargo se han podido identificar algunos elementos que complementan el equipamiento de las casas.

Hogares: se trata de las estructuras más simples dispuestas en el suelo de los espacios de habitación que presentan una superficie rubefactada o ennegrecida por la acción del fuego que endurece la superficie dándole un aspecto característico. Adoptan una forma circular u ovalada y tienen unas dimensiones entre 60 y $80 \mathrm{~cm}$. En ocasiones se apoyan sobre un lecho de cantos rodados y pueden estar delimitados por varias piedras de pequeño tamaño. Están presentes en E2, M1 y M8.

Vasares: son construcciones adosadas a los muros, a veces en un ángulo de la habitación, de aproximadamente un metro de altura, anchura y profundidad variables, erigidas con pequeño aparejo y rellenas de tierra, cuya función parece haber sido la de aparador para los utensilios domésticos o de ocasional banco de trabajo. Los encontramos en M1 y E4.

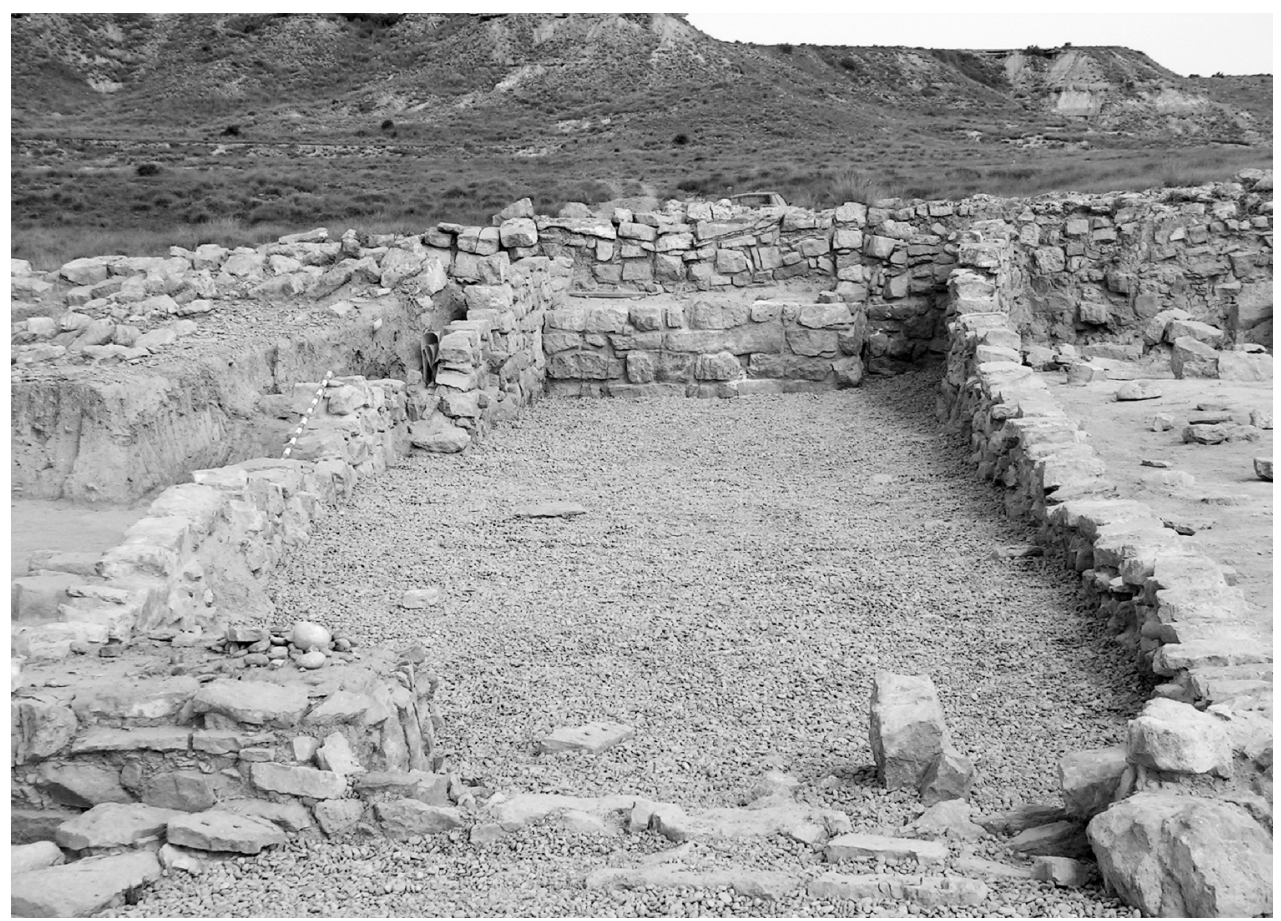

Fig. 9. La habitación M1 con el vasar al fondo y la cubeta junto a la entrada, en primer plano. 
Bancos: Estructuras similares a los vasares, también adosadas a los muros, pero de altura mucho menor, entre 20 y $30 \mathrm{~cm}$., y que parecen haber servido para colocar utensilios o para sentarse en ellos. Han aparecido dos contiguos de distinta altura en M2.

Hornos: Los ejemplares reconocidos hasta el momento se encuentran en un estado de conservación bastante deficiente, conservándose solo la parte inferior mientras que la parte correspondiente a la parrilla y la bóveda ha desaparecido. Están formados por una estructura de planta cuadrada de aproximadamente un metro de lado, aparejadas preferentemente con lajas verticales de tamaño medio, en las que se identifica la boca de la cámara de combustión, formada por un sillar rectangular calzado con dos ripios en ambos lados. Un fragmento de parrilla está construido en cerámica de unos $2 \mathrm{~cm}$. de espesor conservando parte de un orificio circular. Las cámaras debieron ser de barro no habiéndose conservado ni el menor rastro de ellas. Tampoco es posible conocer qué se elaboraba en estos hornos, aunque la presencia de molinos en sus cercanías pudiera interpretarse como un lugar de elaboración de productos relacionados con la molienda de cereal. El espacio E3 está provisto de dos hornos y en E5 se conserva otro.

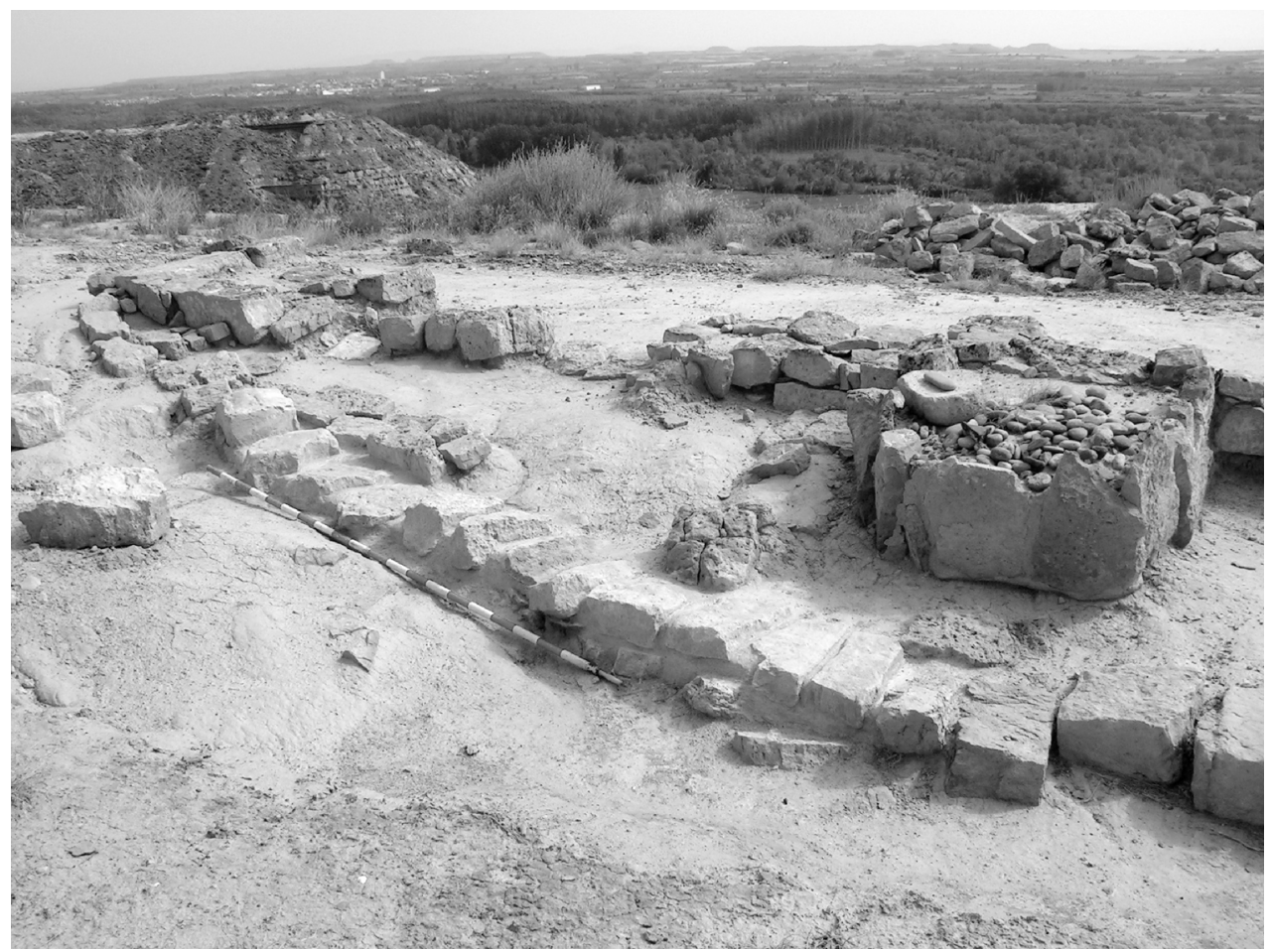

Fig. 10. Horno situado sobre la acera y adosado al espacio E5. 
Enlosados: Estas estructuras, de las que se conserva una completa en E3 y otra parcialmente en E5, están formadas por lajas de pequeño tamaño dispuestas horizontalmente y delimitadas por pequeños sillares. De una altura entre 15 y $20 \mathrm{~cm}$., la completa mide uno por dos metros y ambas se sitúan en un ángulo de la habitación correspondiente. Es interesante destacar que en ambos casos se sitúan próximas a los hornos, lo que hace pensar que su función pudo ser la de leñera o lugar de almacenamiento de otra materia prima (¿grano?). En cualquier caso parecen construidas para proteger los productos de la humedad del suelo.

Tabiques internos: aunque es previsible la construcción de estos elementos domésticos sólo hemos podido documentarlos en dos ocasiones. En M2 se conserva la base de un murete en ángulo formada por pequeños sillares rectangulares cercana a los bancos ya mencionados. El muro divisorio entre los espacios M5 y M6 es otro tabique del que también se conserva únicamente la hilada inferior de pequeños sillares alargados. Desconocemos sus alturas, así como el material del que se elevaron.

Cubetas: Son estructuras que primeramente se identificaron con hogares y cuya función de momento no está clara. Se trata de construcciones de planta

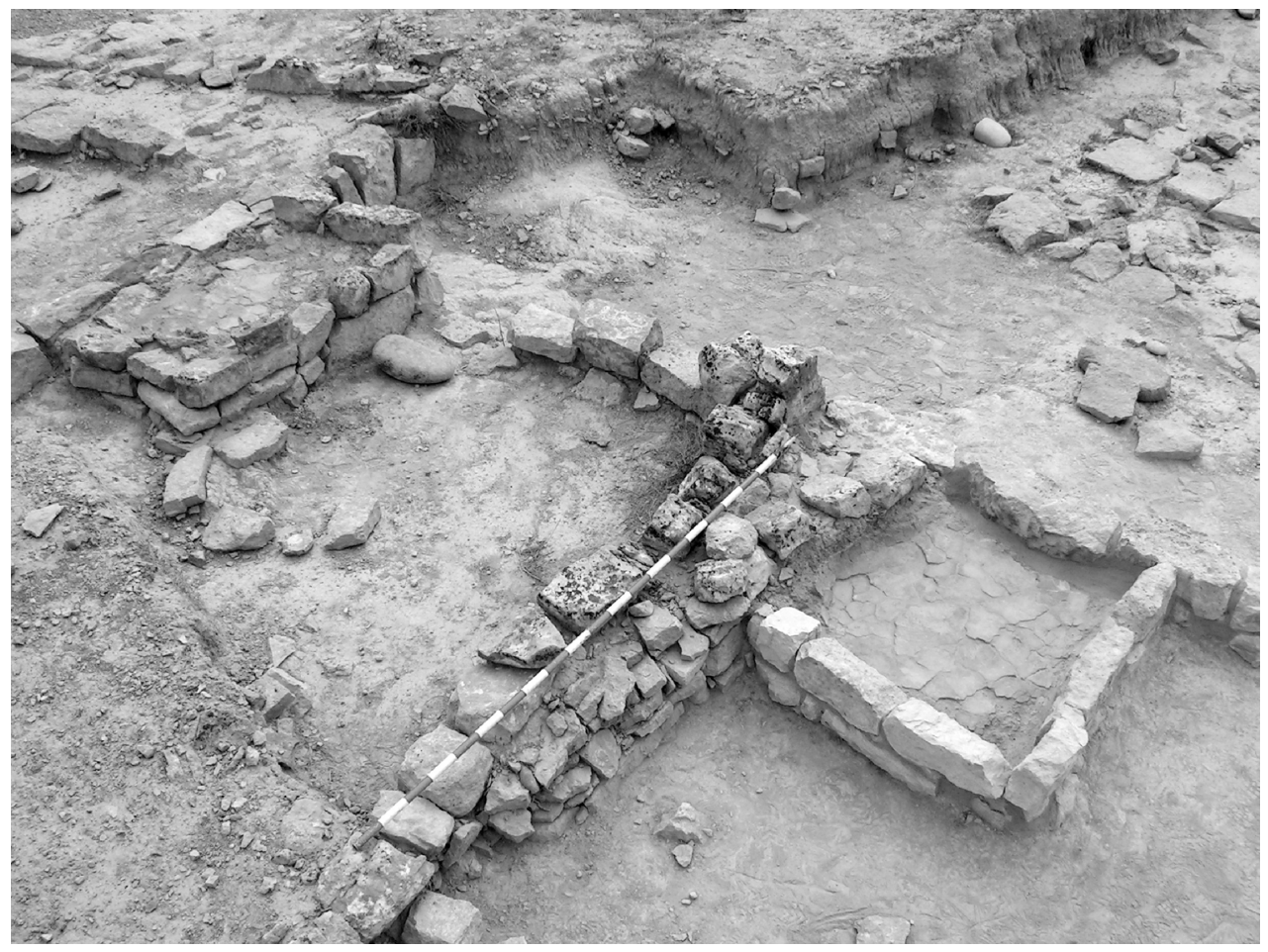

Fig. 11. Cubetas de los espacios M2 (derecha) y M3 (izquierda). 
cuadrada, de un metro de lado, cuya altura conservada es de unos 40 a $50 \mathrm{~cm}$., aparejadas con sillares de pequeño tamaño y lajas colocadas verticalmente. Rellenas de tierra, hasta una altura de unos $40 \mathrm{~cm}$., presentan su superficie superior cubierta de un revestimiento de barro endurecido de escaso espesor $(1 \mathrm{o} 2 \mathrm{~cm}$.) y apoyado sobre un lecho de cantos rodados de pequeño tamaño. En un caso se conserva parte de las paredes que hacen definirlas como cubetas. Al haber desaparecido el pretil de éstas desconocemos la altura (profundidad) que pudieron tener. El todos los casos están adosadas a un ángulo de la habitación, en su parte anterior y junto a la entrada. Se conservan en E2, M1, M2, M3, M5 y E4, todos ellos espacios adosados a la muralla, a excepción de E4, e identificados como viviendas. Su función no está clara pero debe asociarse a alguna actividad de carácter doméstico, relacionada con el almacenamiento o manipulación de materias primas o productos elaborados.

\subsection{Las defensas}

Es evidente que la mejor protección que posee el poblado es su propia ubicación en un lugar alto, fácilmente defendible y de incómodo acceso. La segunda medida de defensa es el recorrido que había que efectuar para acceder al llano, siguiendo desde el río el contorno sur y oeste del espolón ascendiendo por un estrecho barranco que obliga a ofrecer el flanco derecho a los ocupantes del poblado. Pero estas circunstancias se ven notablemente mejoradas por la erección de construcciones de gran envergadura que refuerzan su seguridad.

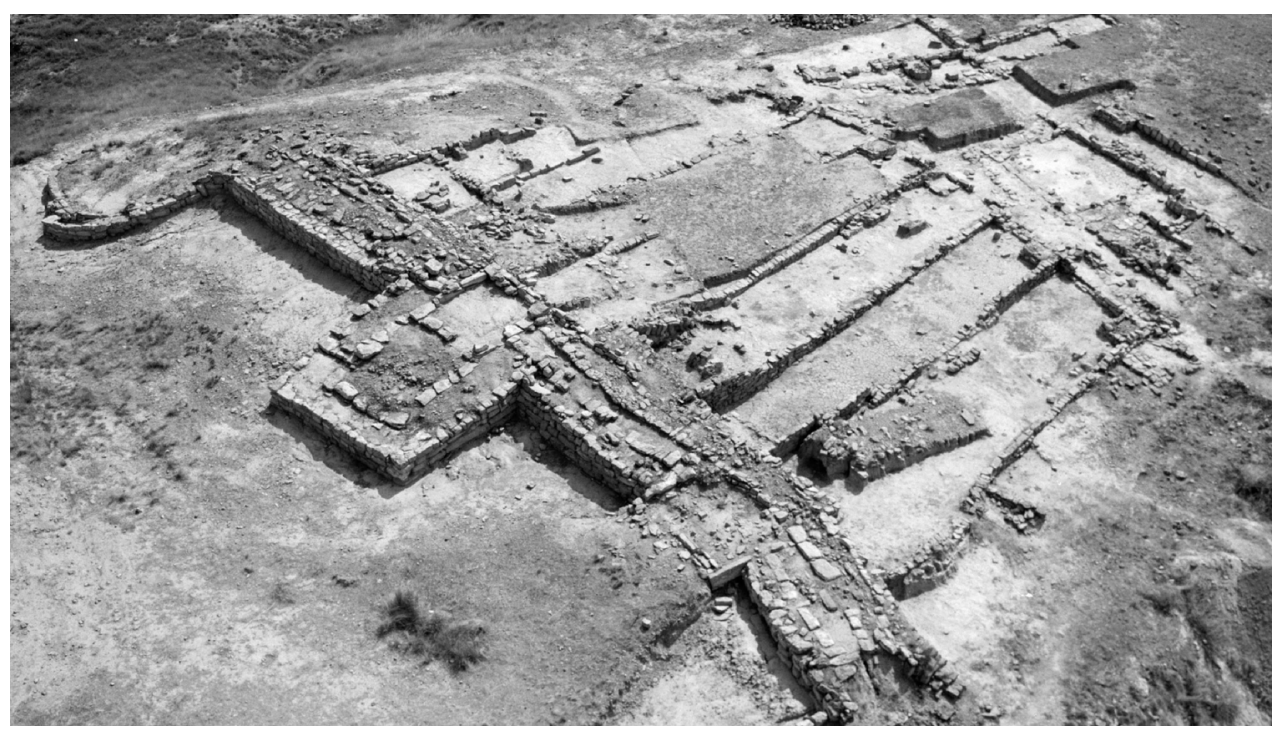

Fig. 12. Vista aérea en la que se aprecia la estructura de la muralla (lienzos, torres y rampa de acceso) y las habitaciones adosadas a ella. 
Ya hemos dicho que la primera construcción defensiva fue un muro que a modo de barrera impedía el acceso al recinto. Este muro está formado por un doble paramento de piedra con un relleno de tierra y piedras y una anchura de unos dos metros; el acceso se situaría en uno de sus lados, probablemente en el extremo occidental por cuestiones defensivas. Sin embargo es en su segunda etapa
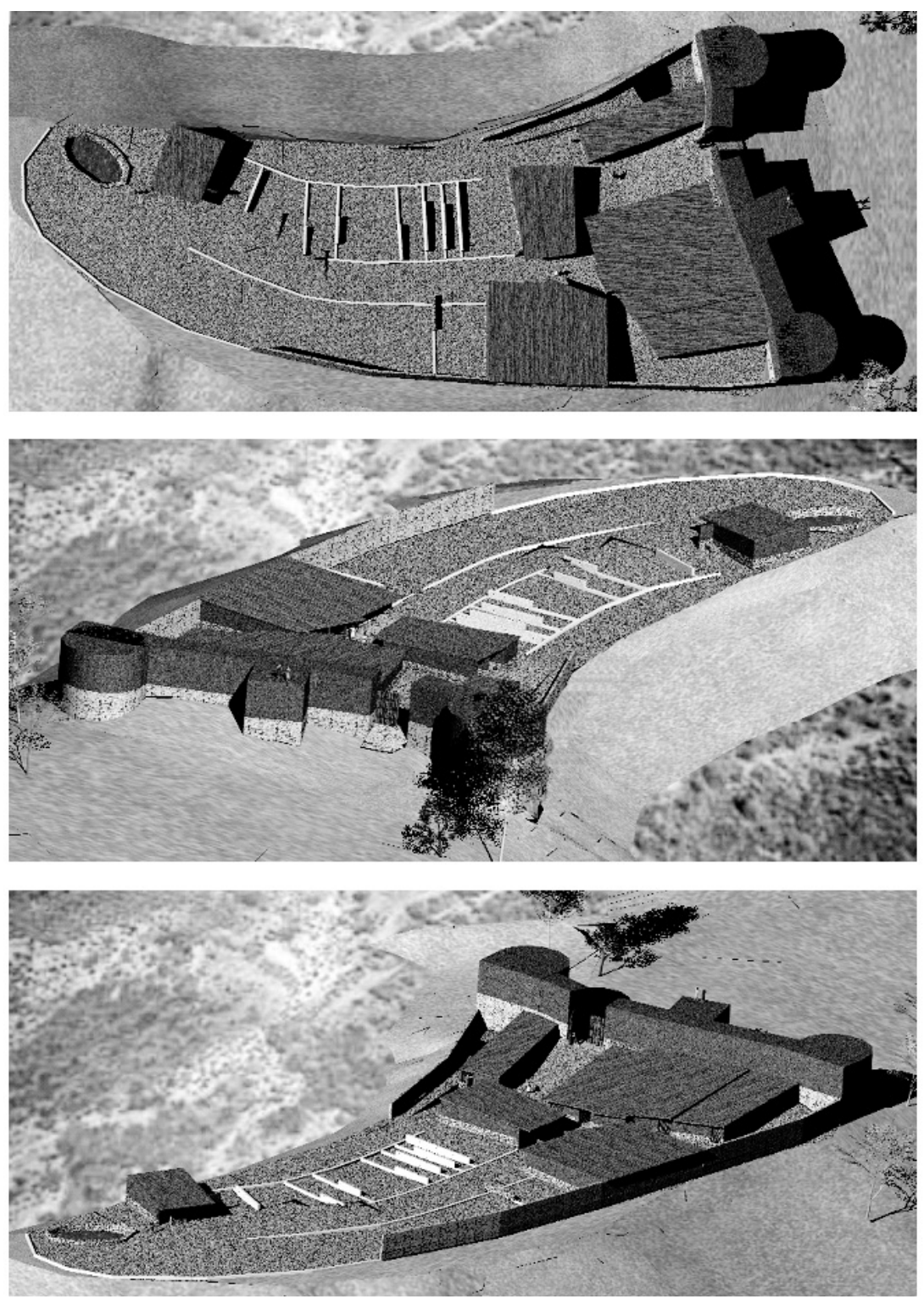

Fig. 13. Propuesta de restitución informática de la última fase de ocupación, según lo excavado hasta 2005. 
cuando se adoptan medidas extraordinarias elevando unas fortificaciones exageradas para las proporciones del poblado. Su función es pues más de prestigio e intimidatoria que consecuencia de una necesidad estratégica.

Primeramente se procedió a un engrosamiento de la muralla añadiendo dos nuevos paramentos en su cara exterior, rellenos de tierra y piedras, a una distancia de un metro, alcanzando así la muralla una anchura cercana a los cuatro metros. El aparejo se acomoda a la topografía original y está formado por sillares de tamaño medio y grande. En el centro de la barrera se levantó una torre cuadrada de unos 7 metros de lado, engastada en los nuevos muros por medio de superposiciones alternativas, de modo que muralla y torre forman un conjunto fuertemente trabado, mucho más resistente que si se hubiera tratado de un simple adosamiento o encastramiento. Esta construcción se hizo con grandes sillares, en este caso muy bien escuadrados. Los extremos de la muralla fueron rematados por sendas torres semicirculares de 10 metros de diámetro.

Al cerrar los extremos de la muralla se condenó la entrada existente y se tuvo que practicar una nueva. El lugar elegido fue un punto intermedio entre la torre central cuadrada y la torre semicircular occidental, favoreciendo su defensa por las torres que la flanquean. Para aumentar el papel defensivo de las torres y proteger todavía más el acceso, la nueva muralla adopta un cambio de dirección formando un ángulo de 170 grados «envolviendo» aún más la puerta propiamente dicha.

Como resultado de estas reformas tuvo que construirse una rampa ante la entrada y en consecuencia una nueva al interior del recinto. Para ello se hubieron de amortizar los espacios E2 y M1 y acondicionar una vía de circulación que conectaba con la calle transversal.

\section{BIBLIOGRAFÍA}

Alonso, N., Garcés, I., Junyent, E., Lafuente, A., López, J., Miró, J. M., Ros, M. T., Rovira, C. (1996): «L'assentament de Els Vilars (Arbeca, les Garrigues): Territori, recursos i activitats productives», Gala, 3-5, pp. 319-339.

Alonso, N., JunYent, E., LAfuente, A., López, J. (1998): «Poder, símbolo y territorio: el caso de la fortaleza de Arbeca», Congreso Internacional Els Ibers, princeps d’Occident, Barcelona, Barcelona, pp. 355-372.

Alonso, N., Junyent, E., LAfuente, A., López, J., TARTERA, E. (2000): «La fortaleza de Arbeca. El proyecto Vilars 2000 ». Investigación, Recuperación y socialización del conocimiento y del patrimonio», Trabajos de Prehistoria, 57, n. ${ }^{\circ}$ 2, Madrid, pp. 161-163.

Domínguez, A., Magallón, M. ${ }^{a}$ A. y Casado, P. (1984): Carta Arqueológica de Huesca, Huesca, pp. 79.

GARCÉS, I., JUNYENT, E. (1989): «Fortificación y defensa en la I Edad del Hierro. Piedras hincadas en Els Vilars», Revista de Arqueología, 93, Madrid, pp. 38-49.

- (1987): «El poblado fortificado de los Campos de Urnas Tardíos en els Vilars, Arbeca, Lleida», XIX Congreso Nacional de Arqueología, Castellón, Zaragoza, vol.Il, 1989, pp.329-339.

GaRCÉs, I., JunYENT, E., LAFUeNTE, A., LÓPEZ, J. (1990): «El sistema defensiu de «Els Vilars» (Arbeca, Les Garrigues)», Actas del Simposi Internacional d'Arqueologia Ibèrica Fortificacions. La problemática de l'iberic ple, Manresa.

JUNYENT, E. (1992): «Els origens del ferro a Catalunya», Arqueologia de Ponent, 2, Lleida, pp.21-35.

JUNYENT, E., LAFUENTE, A., LÓPEZ, J. (1994): «L'origen de l'arquitectura en pedra i l'urbanisme a la Catalunya Occidental», Cota Zero, 10, Barcelona, pp. 73-89. 
MAYA, J. L. (1981): «La Edad del Bronce y la Primera Edad del Hierro en la provincia de Huesca», I Reunión de Prehistoria Aragonesa, Huesca, pp. 129 - 161.

Montón, F. J. (1992): «Las Edades del Bronce y Hierro», en VV. AA: Fraga en la Antigüedad, Zaragoza, pp. 87-132, fig. 16.

- (1998): «Un poblado de la Edad del Hierro en Huesca», Revista de Arqueología 208, Madrid, pp. 60.

- (2001): «La Codera. I Edad del Hierro en el Valle del Cinca (Huesca)», Revista de Arqueología, Madrid, diciembre 2001, pp. 16-23.

- (2003): «Ritual funerario en la I Edad del Hierro. La necrópolis de La Codera», XXVII Congreso Nacional de Arqueología, Huesca, en Bolskan, n. ${ }^{\circ}$ 19, pp. 115-120.

- (e.p.): «La Codera. Hábitat y necrópolis de la I Edad del Hierro», XXVI Congreso Nacional de Arqueología, Zaragoza 2001, Zaragoza.

Página Web: www.lacodera.net

PICAzO, JEsús, y RodANÉS, José M. a (2006): Caminos para el futuro. Ventanas hacia el pasado. El Cabezo de la Cruz, una comunidad agraria de la Edad del Hierro en el Valle del Ebro, Catálogo de la exposición, Zaragoza. 